\title{
Offline rTMS of the primary and premotor cortices does not impact motor sequence memory consolidation despite modulation of corticospinal excitability
}

\author{
Felix Psurek \\ University of Leipzig \\ Bradley Ross King \\ University of Utah \\ Joseph Classen \\ University of Leipzig \\ Jost-Julian Rumpf ( $\sim$ jost-julian.rumpf@medizin.uni-leipzig.de ) \\ University of Leipzig
}

\section{Research Article}

Keywords: motor sequence learning, motor memory consolidation, transcranial magnetic stimulation, corticospinal excitability, premotor cortex, primary motor cortex

Posted Date: July 20th, 2021

DOI: https://doi.org/10.21203/rs.3.rs-646797/v1

License: (c) (i) This work is licensed under a Creative Commons Attribution 4.0 International License.

Read Full License 


\section{Abstract}

Motor skills are acquired and refined across alternating phases of practice (online) and subsequent consolidation in the absence of further skill execution (offline). Both stages of learning are sustained by dynamic interactions within a widespread motor learning network including the premotor and primary motor cortices. Here, we aimed to investigate the role of the dorsal premotor cortex (dPMC) and its interaction with the primary motor cortex (M1) during motor memory consolidation. Forty-eight healthy human participants (age $22.1 \pm 3.1$ years) were assigned to three different groups corresponding to different offline repetitive transcranial magnetic stimulation (rTMS) interventions. $1 \mathrm{~Hz}$ rTMS of either left dPMC, left M1, or sham rTMS was applied immediately after explicit motor sequence training with the right hand. Motor evoked potentials were recorded before training and after rTMS to assess changes of corticospinal excitability (CSE). Participants were retested on the motor sequence after eight hours to assess consolidation. While CSE was not modulated in the sham rTMS group, post-training rTMS increased and decreased CSE when targeting $\mathrm{dPMC}$ and M1, respectively. However, all groups demonstrated similar significant offline learning over the day indicating that offline consolidation was not modulated by the post-training rTMS interventions despite evidence of an interaction of APMC and M1 at the level of CSE. Motor memory consolidation ensuing explicit motor sequence training seems to be a rather robust process that is not affected by rTMS-induced perturbations of $\mathrm{dPMC}$ or M1. Findings further indicate that consolidation of explicitly acquired motor skills is neither mediated nor reflected by posttraining CSE.

\section{Introduction}

The acquisition of a new motor skill is a multi-staged process that evolves both "online", concurrent with repeated skill execution, and "offline", between training sessions [1, 2]. The latter is referred to as motor memory consolidation during which the initially fragile training-induced internal skill model is secured against interference and transformed into a more robust representation in the absence of further skill execution [1, 3-6]. In recent years, a large body of evidence has pointed to a fundamental role of the primary motor cortex (M1) for processing offline motor memory consolidation [2-4, 7-9]. With respect to the neurophysiological mechanisms that may underlie the role of M1 in motor sequence consolidation, Tunovic and co-workers [8] reported that the induction and magnitude of offline-performance increments following motor sequence training was associated with the level of post-training corticospinal excitability (CSE). However, whether the level of post-training excitability of M1 output neurons has a causal and singular role in consolidation of a training-induced motor sequence engram remains an open question. Indeed, a large body of evidence shows that sequential motor skill acquisition is sustained by specific and temporally dynamic interactions between multiple nodes of a widespread neural network that encompasses secondary motor cortical areas, parietal areas, as well as basal ganglia, hippocampus, and the cerebellum in addition to M1 [9-14]. Within this network, recent research has identified the dorsal premotor cortex (dPMC) as a region that (i) has been shown to modulate excitability and plasticity in M1 by interconnections [15-18], (ii) plays an important role in the process of online motor memory formation 
[9, 19-21], and (iii) may thus also be crucially involved in offline motor memory consolidation after motor practice. The latter role is supported by several studies demonstrating modulation of offline motor sequence consolidation by non-invasive brain stimulation (NIBS) of the dPMC [22-27]. However, the interpretation of these previous findings in terms of a crucial role of the $\mathrm{APMC}$ specifically during posttraining motor memory consolidation is difficult, as behavioural effects induced by "excitatory" or "inhibitory" NIBS protocols were inconsistent and the timing of the NIBS intervention in relation to the motor training session (i.e., pre, during, or after) differed across studies.

In the current study, we investigated dPMC and M1 specifically in terms of their role in post-training offline motor memory processing. Findings of previous studies that investigated implicit motor sequence learning suggest that immediate post-training "inhibitory" low-frequency rTMS of the dPMC promotes consolidation [23] while post-training low-frequency rTMS-induced treatment of M1 blocks motor memory consolidation [4]. This may point towards opposing roles of the $\mathrm{dPMC}$ and $\mathrm{M} 1$ during offline motor memory processing after implicit skill acquisition. However, given the evidence that the premotor cortex is particularly critical in explicit acquisition of sequential motor skills [21, 28-31], we applied a purely explicit and ecologically valid motor sequence learning paradigm. Offline (i.e., post-training) lowfrequency repetitive transcranial magnetic stimulation (rTMS) was used to transiently disrupt motor memory processing in the $\mathrm{dPMC}$ and $\mathrm{M} 1$ during the early consolidation process. Effects of stimulation on behavioral markers of consolidation were assessed with a delayed retest administered after an awake interval of eight hours. Additionally, we assessed post-training rTMS-induced changes of CSE to gain insight into the potential interaction of the $\mathrm{dPMC}$ with $\mathrm{M} 1$ during early motor memory consolidation following explicit skill acquisition and to further explore the association of post-training CSE changes with offline consolidation. If malleability of motor memory consolidation by offline NIBS of the APMC and M1 proves to be a robust finding, it would be conceivable to explore its potential as a therapeutic tool to specifically target and modulate motor memory consolidation in the future.

\section{Methods}

\section{Ethical standards}

The study protocol was approved by the institutional ethical standards committee at the University of Leipzig (registration code: 326/18-ek). All methods were performed in accordance with the relevant guidelines and regulations and all participants provided written informed consent before study-related procedures were conducted.

\section{Participants}

Fifty-two young, healthy, and right-handed participants aged between 18 and 30 years (39 female; mean age $22.1 \pm 3.1$ years) were recruited via social media or adverts on the local notice board at the University of Leipzig and completed the experiment. All participants were naïve to the motor sequence learning task and the purpose of the experiment. None of the participants had previously experienced $1 \mathrm{~Hz} r T M S$ or any other type of transcranial magnetic stimulation. Right-handedness was verified using the Edinburgh- 
Handedness-Inventory (EHI, [32]). None of the participants reported a history of neurological or psychiatric disorders (including abuse of alcohol or other illicit drugs) and none of the participants had a relevant medical condition (e.g., rheumatoid arthritis) that might impair task execution. Exclusion criteria further encompassed having been trained as a professional typist or a professional musician to ensure that dexterous finger movements similar to the task employed in our study were not "overlearned". Additional TMS-specific exclusion criteria comprised a history of seizure, central nervous system active medication, and current pregnancy. All participants were further screened for symptoms of depression using the short version of the Beck-Depression-Inventory [33]. The Stanford-Sleepiness-Scale [34] was applied before the initial training session in the morning and the delayed retest session in the evening to assess the possibility that the task execution was confounded by relevant between-group differences in terms of sleepiness/vigilance. Data of four participants were excluded due to insufficient learning of the task as indicated by a negative learning slope across the initial training session (3 participants in the M1 rTMS group, 1 participant in the dPMC rTMS group). The final data set, therefore, comprised experimental data of 48 participants (demographic information is detailed in table 1).

\section{Experimental procedure}

The experiment consisted of a motor sequence training session in the morning and a delayed retest session after eight hours to assess offline performance changes. Participants were randomly assigned to one of three different groups corresponding to three different post-training rTMS interventions (i.e., rTMS applied to the primary motor cortex, M1 group; rTMS applied to the dorsal premotor cortex, dPMC group; sham rTMS, SHAM rTMS group). The post-training $1 \mathrm{~Hz}$ rTMS intervention was applied for 20 minutes immediately following the completion of the initial training session. CSE was assessed by recording TMS-induced motor-evoked potentials from the right abductor pollicis brevis muscle (APB) before the initial training session (MEPpre) and immediately after termination of the post-training rTMS intervention (MEPpost; Fig.1).

\section{Motor sequence learning task}

To assess explicit motor sequence learning, we applied a modified version of the explicit sequential finger-tapping task introduced by Karni and colleagues [7]. Participants were asked to perform a fiveelement finger-tapping sequence (4-1-3-2-4; where $1=$ =index finger, $2=$ middle finger, $3=$ ring finger, $4=$ little finger) on a four-button keyboard with the right hand. Prior to the beginning of the actual training session, participants were required to correctly reproduce the finger-tapping sequence on the keyboard three times in a row to verify explicit knowledge of the sequence. Both the training session and the delayed retest session after eight hours encompassed 14 blocks of successive sequence execution, which were separated by 25-second rest intervals. A green visual cue displayed on a computer monitor in front of the participants indicated an active training block, while a rest period was indicated by a switch of the cue colour to red (i.e., after 60 key taps). Participants were instructed to perform the finger-tapping sequence as fast as possible, while making as few errors as possible. Unbeknownst to the participants, each block 
of active task execution was terminated after 60 key taps, resulting in a maximum number of 12 correct sequences per block. This design ensures that all participants received the same amount of training (i.e., performed the same number of finger movements).

\section{Transcranial Magnetic Stimulation}

Transcranial magnetic stimulation was applied by a MagPro X100 (MagVenture, Farum, Denmark) connected to a MagVenture MC-B70 $70 \mathrm{~mm}$ figure-of-eight coil. The stimulation coil was placed tangentially over the left hemisphere and the handle of the coil was pointed diagonally to the floor behind the subject with an angle of approximately 45 degrees. Electromyography of the right abductor pollicis brevis muscle (APB) was recorded using a Digitimer D360 (Digitimer Ltd., Letchworth Garden, UK). The "motor hotspot" for stimulation of the M1 hand area was identified by applying low-frequency $(<0.2 \mathrm{~Hz})$ stimulation at multiple sites likely overlying left M1 while recording surface EMG from the right APB. We then obtained individual APB resting motor thresholds (RMT) using threshold hunting [35]. BrainSight 2 Neuronavigation (Brain Products, Gilching, Germany) aided to guide constant coil positioning throughout the experiment via virtual landmarks on the scalp. The post-training rTMS intervention encompassed the application of $1200 \mathrm{TMS}$ pulses at a frequency of $1 \mathrm{~Hz}$ (i.e., 20 minutes of stimulation) with the stimulator output intensity set to $110 \%$ of the individual RMT. For the post-training rTMS intervention in the M1 group, the coil was placed over the individual "motor hotspot", whereas post-training rTMS of the dorsal premotor cortex in the dPMC group was applied with the similarly oriented coil placed $2.5 \mathrm{~cm}$ anterior of the APB "motor hotspot" $[16,17]$. In the SHAM group, rTMS was applied at the same frequency and stimulator output intensity, but the coil was placed vertically to the scalp approximately over M1. This procedure results in the characteristic TMS-sound while providing no effective stimulation. During the post-training stimulation period, participants were asked to remain seated as still as possible with their eyes open. Coil positioning and orientation to stimulate M1 or $\mathrm{dPMC}$ were kept constant by using the neuro-navigation system. APB surface EMG was recorded across the post-training stimulation period to verify that the right hand was relaxed, to assess potential changes of APB MEP amplitude sizes across time in the M1 group and to demonstrate that post-training dPMC rTMS did not induce suprathreshold M1 stimulation caused by current spread due to the proximity of stimulation sites. To assess potential rTMS-induced modulation of corticospinal excitability (CSE), 20 APB-MEPs that were elicited at $0.1 \mathrm{~Hz}$ with the stimulator output set to an individually adjusted intensity to produce MEPs of $\sim 1 \mathrm{mV}$ were recorded before introduction of the task and onset of training (MEPpre) and immediately following the post-training rTMS intervention (MEPpost; Fig. 1).

\section{Data Acquisition and Analysis}

Custom MatLab (Mathworks, Natick, USA) scripts were used to record the timing of key presses and to extract measures of speed performance and accuracy. Speed was defined as the average time (seconds) needed to execute correct sequences within a given block of task training (correct sequence duration, CSD). Accuracy was defined as the ratio of the number of correctly performed sequences per block 
divided by the maximum number of correct sequences per block (i.e., 12). To take potential interindividual differences into account with respect to the strategy to improve task performance (e.g., prioritize speed over accuracy, or vice versa), we additionally quantified task performance using a performance index (PI) that incorporates both equally important components of task performance [36].

\section{$P I x=100 * e^{-C S D x} * e^{A C C x-1}$, where $\mathrm{x}=$ block of trials.}

Effects of repeated practice on task performance across the initial training session and the delayed retest session (i.e., online performance changes) were assessed by applying separate repeated measures analyses of variance (rmANOVA) to the training and retest speed, accuracy, and combined PI values with Group (post-training M1, dPMC, SHAM rTMS) as the between-subject factor and Block (e.g., B1, ..., B14) as within-subject factor. This allowed us to investigate potential between-group differences in terms of the magnitude and rate of online performance changes as a function of repeated practice within the training and delayed retest sessions. Offline consolidation effects were computed as the difference between the individual end-of-training baseline (EoT; i.e., average performance across the last 4 blocks of the training session) and the performance at the beginning of the delayed retest (BoRT, performance in the first block of the delayed retest) so that positive values indicate offline performance increments relative to EoT and negative values indicate offline performance impairments. We chose to use only the first block of retesting to compute offline consolidation to not confound the consolidation measure with additional online learning.

Electromyographic data was recorded using "CED Signal" (Cambridge Electronic Design Ltd., Cambridge, England) and manually processed with the "palMEP" tool (Perellón-Alfonso et al. 2018). All recorded MEPs were evaluated separately and discarded if any artefacts (pre-activation, voluntary movements etc.) were observed in the EMG recordings. For evaluation of potential rTMS-induced effects on CSE, we averaged MEP amplitude sizes across MEPpre and across MEPpost assessments and applied a rmANOVA with the within-subject factor Time (MEPpre, MEPpost) and the between-subject factor Group to these values. To assess alterations of CSE across the 20 minutes of the post-training $1 \mathrm{~Hz}$ rTMS intervention in the M1 group, MEP amplitude sizes were averaged across blocks of 4 minutes (240 frames) each and analysed using rmANOVA with the within-subject factor Time (minutes 1-4, 5-8, 9-12,13$16,17-20)$.

Spearman's rank correlation coefficient was applied to assess associations of rTMS-induced modulation of CSE (difference between MEPpost and MEPpre; MEPpost) and the magnitude of offline performance changes.

All statistical analyses were performed with SPSS 25 (SPSS, Chicago, IL, USA). For all statistical tests, the alpha level was set to $p<0.05$. rmANOVAs were checked for violation of sphericity and degrees of freedom and p-values were corrected accordingly if necessary (Greenhouse-Geisser correction). 


\section{Results}

\section{Participant characteristics}

Demographic information and characteristics of the three groups (M1, dPMC, SHAM rTMS) corresponding to the three different post-training rTMS interventions are detailed in table 1. There were no significant between-group differences in terms of age, sex, depressive symptoms as assessed by the BDI, handedness as assessed with the EHI, the baseline resting motor threshold, nor the stimulation intensity required to elicit MEPs of approximately $1 \mathrm{mV}$ (all $\mathrm{p} \geq 0.114$ ). There were also no significant differences in terms of vigilance before training $(p=0.161)$, and before the delayed retest $(p=0.265)$ as assessed with the StanfordSleepiness-Scale. This collectively indicates that potential between-group differences in terms of online and offline motor learning are unlikely to be generated by differences in group demographics and characteristics.

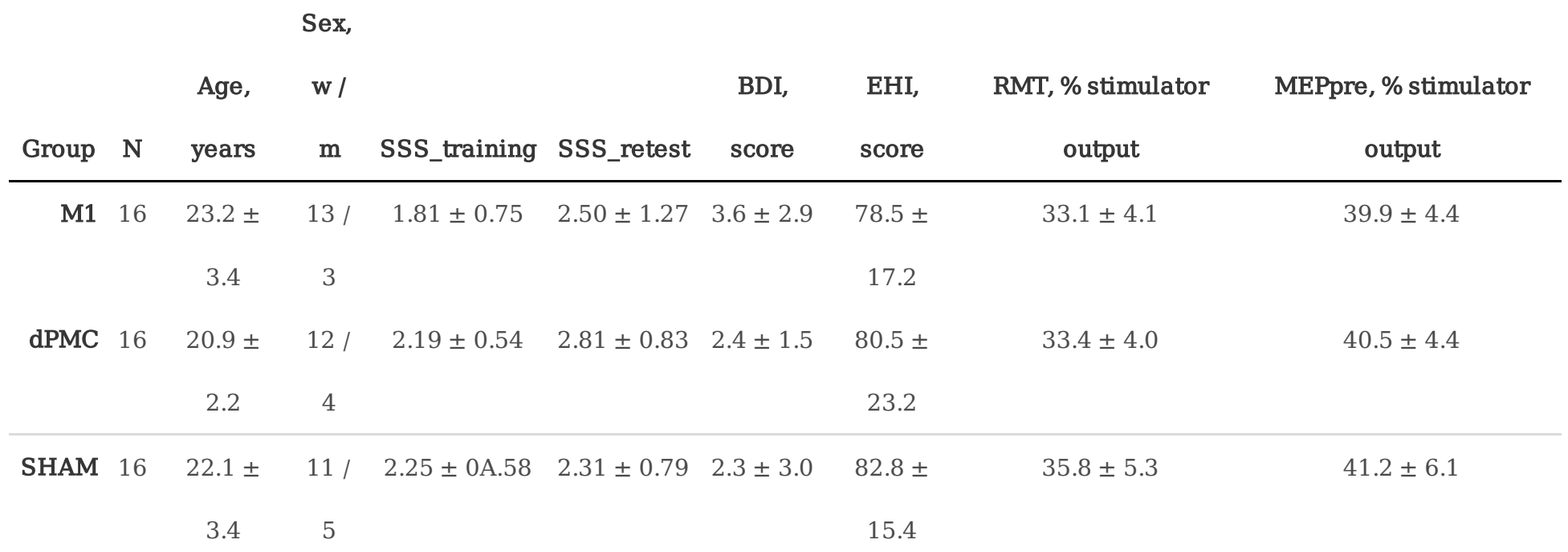

Table 1: Group characteristics. Stanford-Sleepiness-Scale score before training (SSS_training) and before retest (SSS_retest); N, number of participants; BDI, Beck-Depression-Inventory; EHI, Edinburgh-Handedness-Inventory; RMT, Resting Motor Threshold; MEPpre, individual stimulation intensity to elicit MEPs of $\sim 1 \mathrm{mV}$ at baseline. All values (excluding sex and number of participants) are displayed as mean \pm standard deviation.

\section{Post-Training rTMS Effects on Corticospinal Excitability}

Pre-training baseline CSE as indexed by average MEP amplitude was $0.91 \mathrm{mV}$ (CI 0.73 - 1.09) in the M1 rTMS group, $0.93 \mathrm{mV}$ (CI 0.57 - 1.28) in the dPMC rTMS group, and $0.88 \mathrm{mV}$ (CI 0.59 - 1.16) in the SHAM rTMS group and, thus, did not significantly differ between groups $\left(\mathrm{F}_{(2,45)}=0.037, \mathrm{p}=0.964\right)$. A rmANOVA including the between-subject factor Group (M1, dPMC, 
SHAM) and the within-subject factor Time (MEPpre, MEPpost) revealed a significant main effect of Group $\left(\mathrm{F}_{(2,45)}=5.898, \mathrm{p}=0.005\right)$ and a significant Time $x$ Group interaction $\left(F_{(2,45)}=7.684, p=0.001\right)$ in the absence of a main effect of Time $\left(F_{(1,45)}=1.588, p=0.214\right)$. Subsequent rmANOVA showed that the significant interaction of Time x Group was driven by a significant decrease of the average MEPpost amplitude compared to the average MEPpre amplitude in the M1 rTMS group $\left(-0.337 \mathrm{mV}\right.$, CI $-0.566-0.109$, Time: $\mathrm{F}_{(1,15)}=9.886$, $\mathrm{p}=0.007$ ), while there was a significant MEP amplitude increase in the dPMC rTMS group (+0.868 mV, CI $0.202-1.535$; Time: $\left.\mathrm{F}_{(1,15)}=7.706, \mathrm{p}=0.014\right)$, and no relevant change of CSE in the SHAM group $\left(-0.037 \mathrm{mV}\right.$, CI -0.486 - 0.412; Time: $\mathrm{F}_{(1,15)}=0.031, \mathrm{p}=0.863$; Fig. 2). Collectively, this demonstrates that post-training CSE was significantly and differentially modulated depending on the type/location of the post-training rTMS intervention. While, as expected, post-training $1 \mathrm{~Hz}$ M1 rTMS significantly decreased the size of the MEP amplitudes, post-training $1 \mathrm{~Hz}$ rTMS of the dPMC significantly increased CSE compared to the pre-training assessment. Evaluation of the evolution of CSE across the post-training $1 \mathrm{~Hz}$ rTMS intervention directed to M1 showed that the MEP amplitude size decreased from an average of $0.382 \mathrm{mV}$ (CI 0.017 - 0.746) during the first four minutes to $0.245 \mathrm{mV}$ (CI 0.088 - 0.402; rmANOVA Time: $\left.\mathrm{F}_{(1.15,16.13)}=1.558, \mathrm{p}=0.234\right)$ across the last four minutes of stimulation (please note that data of one participant were excluded from this ANOVA due to missing data at the beginning of the post-training rTMS stimulation period). No MEPs were detected during the post-training $1 \mathrm{~Hz}$ SHAM rTMS intervention or during the $1 \mathrm{~Hz}$ dPMC stimulation. The latter obeservation demonstrates absence of supra-threshold current spread despite the proximity of the dPMC stimulation site to M1.

\section{Behavioural results}

Accuracy

Accuracy of task performance was very high amounting to 0.937 (CI $0.924-0.951$ ) across the training and delayed retest sessions, indicating that participants, on average, produced less than one incorrect sequence per block (Fig. 3A). Moreover, a rmANOVA conducted on the accuracy measure revealed no significant effect for the factors Group (M1, dPMC, SHAM rTMS) and Block (B1, ..., B14), or the interaction of both factors across the initial training session (all $p \geq 0.385$ ) as well as across the delayed retest session (all $p \geq 0.117$ ). Collectively, this 
indicates that motor sequence performance in terms of accuracy was not modulated by repeated task practice, potentially because participants performed at or close to accuracy ceiling even at the beginning of the experiment. Online task learning and potential effects of the rTMS intervention on offline consolidation were therefore assessed using speed as the primary performance measure.

\section{Training Session - Online Learning}

A rmANOVA conducted on the speed performance measure (CSD) with the between-subject factor Group (M1, dPMC, SHAM rTMS) and the within-subject factor Block (B1, ..., B14) revealed a significant main effect of $\operatorname{Block}\left(\mathrm{F}_{(3.79,170.35)}=54.745, \mathrm{p}<0.001\right)$ in the absence of a significant main effect of Group $\left(\mathrm{F}_{(2,45)}=0.011, \mathrm{p}=0.989\right)$, or a significant interaction of both factors $\left(\mathrm{F}_{(7.57,170.35)}=0.763, \mathrm{p}=0.629\right)$. Average baseline CSD in the first block of the training session amounted to 1.624 s (CI 1.480 - 1.769) and did not significantly differ between groups $\left(F_{(2,45)}=0.038, p=0.963\right)$. Speed performance reached a similar asymptotic plateau in all groups at the end of the training session (EoT, average performance across the last 4 blocks of the training session; 1.068 s, CI 0.977-1.160), as indicated by a non-significant effect of Block $\left(\mathrm{F}_{(2.45,110.45)}=1.669, \mathrm{p}=0.186\right)$ and Group $\left(\mathrm{F}_{(2,45)}=0.003, \mathrm{p}=0.997\right)$, and the absence of a significant interaction of Block x Group $\left(\mathrm{F}_{(4.91,110.45)}=1.164, \mathrm{p}=0.332\right)$. The above results, collectively, indicate that participants in all groups increased speed of performance at a similar rate across the initial training session and reached similar asymptotic performance at the end of the training session (EoT), against which consolidation effects were assessed. This rules out that potential effects of the following offline rTMS intervention on consolidation may have been confounded by differences of online skill acquisition during the training session.

\section{Delayed Retest - Offline Consolidation}

Offline performance changes as measure of consolidation were assessed between EoT (average performance across the last 4 blocks of training) and the performance at the beginning of the delayed retest (BoRT, first block of delayed retesting). rmANOVA including the within-subject factor Time (EoT, BoRT) and the between-subject factor Group revealed a significant main

effect of the factor Time $\left(\mathrm{F}_{(1,45)}=8.605, \mathrm{p}=0.005\right)$, which was driven by an offline speed (CSD) improvement between EoT and BoRT of 63.8 ms (CI 20.9 - 106.6) across all groups. There was no significant main effect of the factor Group $\left(\mathrm{F}_{(2,45)}=0.005, \mathrm{p}=0.995\right)$, and, most importantly, 
no significant interaction of Group $\mathrm{x}$ Time $\left(\mathrm{F}_{(2,45)}=0.162, \mathrm{p}=0.851 ;\right.$ Fig. 3B). This indicates that, although $1 \mathrm{~Hz}$ rTMS of M1 and $1 \mathrm{~Hz}$ rTMS of dPMC significantly modulated post-training CSE in different directions, neither type of intervention had a relevant effect on the consolidation process compared with the sham intervention. Of note, we had decided to use the first block of retesting to compute offline performance changes against EoT to not confound the consolidation measure with additional task training and potential online learning effects. However, the above results remain similar if averages of different subsets of delayed retest blocks (e.g., average of the first two, three, or four retest blocks) were used to compute the consolidation measure.

We further assessed whether the post-training rTMS intervention may have affected potential differences in online learning across the delayed retest session by applying a rmANOVA on the CSD values across the 14 blocks of delayed retesting. This analysis revealed a significant main effect of Block $\left(\mathrm{F}_{(6.37,286.74)}=7.700, \mathrm{p}<0.001\right)$, while there was no significant main effect of Group $\left(\mathrm{F}_{(2,45)}=0.209, \mathrm{p}=0.812\right)$, nor a significant interaction of Group $\mathrm{x}$ Block $\left(\mathrm{F}_{(12.74,286.74)}=1.155, \mathrm{p}=0.314\right)$, indicating similar online learning across the delayed retest session independent of the previous post-training rTMS intervention. In addition to the observation of significant offline performance improvements from EoT to BoRT across all groups, the significant Block effect in this rmANOVA suggests that the lack of a between-group difference in offline consolidation cannot be attributed to reaching a ceiling in performance speed at the end of the initial training session. In accordance with the above results, nonparametric correlation analyses revealed no relevant association of the magnitude of offline performance changes between EOT and BoRT with the amplitude of the MEPpost amplitudes ( $\mathrm{r}$ $=0.139, \mathrm{p}=0.346$ ) nor with the magnitude of rTMS-induced changes of CSE (Delta MEPpost MEPpre; $\mathrm{r}=-0.055, \mathrm{p}=0.713$ ).

Performance Index

Although average accuracy across blocks and groups was not relevantly modulated by the task, we chose to additionally apply a performance index incorporating speed and accuracy measures to account for potential interindividual differences with respect to the strategy to improve task performance (e.g., prioritize speed performance at the expense of accuracy). A rmANOVA conducted on the PI values across the training session revealed a significant main effect of Block $\left(\mathrm{F}_{(6.08,272.77}=41.049, \mathrm{p}<0.001\right)$ in the absence of a significant effect of Group 
and absence of a significant interaction of Group $x$ Block (both $\mathrm{p} \geq 0.837$ ), indicating similar online learning across the training session (Fig. 3A) Initial average PI of the first block of training amounted to 21.38 (CI 18.43 - 24.33) and reached an average of 34.04 (CI 31.08 36.99) across the last four blocks of training (EoT). Similar to the speed performance analysis, a rmANOVA with the within-subject factor Time (EoT, BoRT) and the between-subject factor Group was conducted to assess potential differences in offline consolidation. While there was a significant effect of the main factor Time $\left(\mathrm{F}_{(1,45)}=14.948, \mathrm{p}<0.001\right)$, this analysis revealed no significant effect of Group nor a significant interaction of factors Time x Group (both $\mathrm{p} \geq 0.483$ ). The significant main effect of time was driven by an offline average PI improvement between EoT and BoRT of 2.81 (CI 1.36 - 4.27) across all groups (Fig. 3B), indicating significant offline learning during the consolidation period irrespective of the type of post-training rTMS intervention (again, results were similar when different subsets of retest blocks were defined as BoRT). Performance increased further across the 14 delayed retest session blocks in all groups as indicated by a significant effect of $\operatorname{Block}\left(\mathrm{F}_{(8.14,366.37)}=2.665, \mathrm{p}=0.007\right)$, while there was no significant main effect of Group or a significant interaction of Block x Group (both $p \geq 0.625$ ), demonstrating that also online learning during delayed retesting was not modulated by any type of the prior post-training rTMS intervention. Moreover, there were no significant correlations of the magnitude of offline performance changes between EOT and BoRT with the amplitude of the MEPpost amplitudes $(r=0.071, \mathrm{p}=0.630)$ or the magnitude of rTMSinduced changes of CSE (Delta MEPpost - MEPpre; $r=-0.044, p=0.766$ ). Consistent with the analyses of movement speed above, these results collectively indicate that the stimulation intervention had no impact on motor sequence performance as assessed with a speed-accuracy aggregate measure.

\section{Discussion}

The current study was primarily designed to investigate the function of the premotor cortex and its potential interaction with the primary motor cortex during early post-training motor memory consolidation. Our main findings were that targeting the dPMC with "inhibitory" rTMS immediately after explicit motor sequence training enhanced the excitability of M1 output neurons but had no modulatory effect on subsequent offline skill consolidation over the day. As expected, post-training $1 \mathrm{~Hz}$ rTMS applied to $M 1$ induced a significant decrease of post-training CSE. However, post-training rTMS of M1 also failed to induce a relevant behavioural effect on subsequent motor memory consolidation. Importantly, online learning across the initial training session was similar among all three experimental groups (i.e., post- 
training sham rTMS, dPMC rTMS, and M1 rTMS) with respect to all assessed measures of performance (i.e., speed, accuracy, and the combined performance index). This rules out that potential post-training rTMS-induced effects on consolidation or CSE were confounded by differences of online skill acquisition within the initial training session. Moreover, all three experimental groups demonstrated similar significant between-session performance increments over the day (i.e., offline-learning) independent of the level of post-stimulation CSE or CSE changes between the pre-training baseline and the poststimulation assessments. Collectively, the present findings are at odds with the idea that any role that the dPMC or M1 might have in the early consolidation phase of explicitly acquired sequential motor skills can be disrupted by post-training rTMS. Furthermore, although our findings do not exclude a role of dPMC or M1 in early consolidation, it is highly unlikely that this role is mediated by, or is apparent as changes of CSE.

Previous studies that investigated the role of the premotor cortex in motor learning reported that the posttraining consolidation process may be modulated by NIBS of the $\mathrm{APMC}$, indicating its relevant involvement in motor memory consolidation. However, results of these studies are inconsistent in terms of the direction of effects on motor memory consolidation induced by the application of either "excitatory" or "inhibitory" NIBS protocols. While Boyd and co-workers [22] reported a facilitation of motor memory consolidation by application of "excitatory" $5 \mathrm{~Hz}$ rTMS to the dPMC (and no effect of $1 \mathrm{~Hz} \mathrm{rTMS}$ ), Kantak and co-workers [24] demonstrated impaired motor consolidation when the dPMC was targeted with "excitatory" anodal tDCS. On the other hand, application of "inhibitory" cathodal tDCS to the dPMC resulted in impaired delayed reproduction of a motor sequence in one study [26], while the same intervention was reported to facilitate post-training stabilization of a newly learned motor sequence in another study [25]. Moreover, the interpretation of these findings in terms of a role of the dPMC specifically during early offline motor-memory consolidation is difficult because in the above studies NIBS was applied either before [22, 25, 26], or during [24] task training. Therefore, it cannot be excluded that the reported effects on subsequent consolidation were confounded by an interaction of NIBS-induced effects on dPMC (or persisting after-effects if applied before training) with processing of online skill acquisition during training. However, studies in which NIBS of dPMC was applied after motor sequence training - thus exclusively targeting the direct interaction of NIBS-induced modulation of dPMC with early post-training offline motor memory processing - also produced mixed results. While Meehan and coworkers [23] reported facilitation of motor sequence consolidation by immediate post-training "inhibitory" $1 \mathrm{~Hz}$ rTMS of the dPMC, which might suggest an inhibitory influence of the dPMC during early consolidation, Nitsche and co-workers [27] demonstrated enhanced consolidation by delayed posttraining "excitatory" anodal tDCS of the dPMC during sleep. However, immediate post-training "excitatory" anodal tDCS of the dPMC exerted no effect on motor sequence consolidation in a group of healthy older people in a recent study from our group [37]. Findings of the latter study are in line with the current results that do not provide evidence of a relevant involvement of the $\mathrm{dPMC}$ during early post-training consolidation following explicit training-induced sequential motor skill acquisition in young adults. This interpretation may be supported by a recent meta-analysis that suggests that motor sequence learning is mainly and particularly driven by contributions of the basal ganglia, whereas the premotor cortex as well 
as the cerebellum play a subordinate or negligible role for learning-specific aspects in motor sequence learning [38]. Collectively, if the above body of evidence indeed reflects absence of the dPMC in motor sequence learning, then this interpretation may perhaps explain the inconsistent results of the dPMC NIBS studies.

Another potential explanation may be that the diverging results of the APMC NIBS studies are driven by different task characteristics in terms of explicit or implicit motor sequence skill acquisition. Explicit and implicit sequence learning are believed to be sustained by at least partly different neural substrates and involvement of the premotor cortex was reported to be particularly critical in explicit sequence learning $[21,28,29]$. However, in the current study as well as in our previous study [37], which both indicated no relevant role of the $\mathrm{dPMC}$ during early consolidation, we used a purely explicit motor sequence learning task. While this does not exclude a role of the $\mathrm{dPMC}$ during explicit online learning, it still questions a relevant role of the $\mathrm{dPMC}$ at least during early consolidation of explicitly acquired sequential motor skills. Moreover, findings in terms of involvement of the dPMC during consolidation were also inconsistent among the studies that applied implicit serial reaction time tasks [23-25, 27]. Collectively, this body of evidence does not support the conclusion that involvement of the dPMC during post-training consolidation is determined by whether motor sequence skill was acquired under explicit or implicit conditions.

As expected, and consistent with earlier research [39-41], $1 \mathrm{~Hz}$ rTMS of M1 decreased CSE across the posttraining intervention, resulting in significant CSE suppression in the post-stimulation (MEPpost) assessment. Interestingly, post-training $1 \mathrm{~Hz}$ rTMS of the dPMC induced the opposite effect on CSE, i.e., significant facilitation of post-training CSE. Besides the fact that we did not detect any MEPs during the post-training $\mathrm{dPMC}$ rTMS intervention, this excludes suprathreshold current spread from the dPMC stimulation to M1 which would likely have resulted in decreased CSE. One might then still ask whether facilitation of CSE by $1 \mathrm{~Hz}$ rTMS of the dPMC may be caused by subthreshold current spread to M1. However, we think that this is unlikely as we are not aware of any studies that suggest facilitation of CSE by subthreshold low-frequency M1 rTMS. Thus, facilitation of CSE by post-training $1 \mathrm{~Hz}$ rTMS of dPMC rather indicates modulation of $\mathrm{M} 1$ output neuron excitability via $\mathrm{dPMC}>\mathrm{M} 1$ projections. This interpretation may be supported by previous studies that also demonstrated modulation (albeit depression) of CSE by low-frequency rTMS $[17,42]$ or continuous theta burst stimulation $[15,16]$ of the $\mathrm{dPMC}$ in the absence of a prior motor training intervention. Facilitation of M1 output neuron excitability by post-training inhibitory rTMS of $\mathrm{dPMC}$ in the current study points to the possibility of a tonic inhibitory input of the $\mathrm{dPMC}$ on $\mathrm{M} 1$ during early consolidation after explicit sequence skill acquisition that reflects a state-dependent interconnection of M1 and dPMC. This view is, however, challenged by findings of Meehan and co-workers [23] who did not report relevant changes of CSE following post-training lowfrequency rTMS of dPMC after implicit motor sequence learning.

Another interesting aspect of our findings was that also targeting M1 with low-frequency rTMS after motor sequence training did not affect consolidation despite rTMS-induced reduction of post-training CSE. This was unexpected given previous findings suggesting that immediate post-training inhibition of 
M1 by $1 \mathrm{~Hz}$ rTMS interventions was shown to not only block offline consolidation of simple ballistic finger movement skills [3], but to also block over-the-day consolidation of more complex implicitly-acquired finger tapping sequences in a study by Robertson and co-workers [4]. The induction of consolidation over the day was, furthermore, linked to post-training CSE by Tunovic and colleagues [8] who reported that acquisition of a sequential motor skill under explicit conditions induced an immediate post-training decrease of CSE which was associated with absence of offline-learning over the day, while performing the same task under implicit conditions did not induce a post-training CSE decrease and was followed by significant offline performance gains. Moreover, the magnitude of offline-performance increments over the day was shown to be associated with the level of immediate post-training CSE. Interestingly, the induction of offline performance gains over the day subsequent to explicit task training was restored when the immediate post-training CSE decrease was prevented by remote rTMS [8]. Facilitation of consolidation following training-induced acquisition of sequential motor skills by post-training application of anodal transcranial direct current stimulation (tDCS) of M1 [37, 43, 44] may, thus, be explained by a similar mechanism, i.e., facilitation of immediate post-training CSE. Collectively, these observations have been suggested to indicate that the level of immediate post-training CSE represents a neurophysiological signal that determines whether and how much training-induced motor representations are promoted offline over the day or not. However, results with respect to facilitation of consolidation by offline application of tDCS to M1 are inconsistent for both explicit [45] and implicit motor sequence learning [46]. Moreover, we did not detect an immediate post-training decrease of CSE as described by Tunovic and co-workers [8], although a purely explicit motor sequence learning task was applied in the current study. Moreover, neither subsequent post-training depression of CSE induced by M1 rTMS nor significant remote facilitation of post-training CSE by dPMC rTMS influenced the magnitude of offline skill performance changes between sessions. This suggests that neither (i) dichotomic differential modulation of immediate post-training CSE by prior implicit or explicit sequential motor skill acquisition nor (ii) determination of induction of consolidation by the level of post-training CSE are generalizable principles in motor sequence learning.

In conclusion, despite evidence of an interaction of $\mathrm{dPMC}$ and M1 at the level of CSE, current findings indicate that the post-training consolidation process following motor sequence skill acquisition under explicit conditions is not accessible to perturbation of dPMC or M1 by rTMS. In contrast to previous findings that suggest modulation of motor memory consolidation by post-training NIBS of dPMC [23] and M1 [4] in implicit motor sequence learning, this may suggest that offline processing of explicitly acquired motor sequence skills is a rather robust process that is at least not malleable by rTMS-induced manipulation of just single nodes of the motor learning network. Our results further indicate that offline processing of explicitly acquired motor sequences is neither mediated nor reflected by post-training CSE changes which questions the generalizability of this marker as a predictor of consolidation beyond specific learning tasks.

\section{References}


1. Dayan, E., and Cohen, L.G. (2011). Neuroplasticity subserving motor skill learning. Neuron 72, 443454.

2. Karni, A., Meyer, G., Rey-Hipolito, C., Jezzard, P., Adams, M.M., Turner, R., and Ungerleider, L.G. (1998). The acquisition of skilled motor performance: fast and slow experience-driven changes in primary motor cortex. Proc Natl Acad Sci U S A 95, 861-868.

3. Muellbacher, W., Ziemann, U., Wissel, J., Dang, N., Kofler, M., Facchini, S., Boroojerdi, B., Poewe, W., and Hallett, M. (2002). Early consolidation in human primary motor cortex. Nature 415, 640-644.

4. Robertson, E.M., Press, D.Z., and Pascual-Leone, A. (2005). Off-line learning and the primary motor cortex. J Neurosci 25, 6372-6378.

5. Janacsek, K., and Nemeth, D. (2012). Predicting the future: from implicit learning to consolidation. Int J Psychophysiol 83, 213-221.

6. Doyon, J., Bellec, P., Amsel, R., Penhune, V., Monchi, O., Carrier, J., Lehericy, S., and Benali, H. (2009). Contributions of the basal ganglia and functionally related brain structures to motor learning. Behav Brain Res 199, 61-75.

7. Karni, A., Meyer, G., Jezzard, P., Adams, M.M., Turner, R., and Ungerleider, L.G. (1995). Functional MRI evidence for adult motor cortex plasticity during motor skill learning. Nature 377, 155-158.

8. Tunovic, S., Press, D.Z., and Robertson, E.M. (2014). A physiological signal that prevents motor skill improvements during consolidation. J Neurosci 34, 5302-5310.

9. Hardwick, R.M., Rottschy, C., Miall, R.C., and Eickhoff, S.B. (2013). A quantitative meta-analysis and review of motor learning in the human brain. Neuroimage 67, 283-297.

10. Albouy, G., Sterpenich, V., Balteau, E., Vandewalle, G., Desseilles, M., Dang-Vu, T., Darsaud, A., Ruby, P., Luppi, P.H., Degueldre, C., et al. (2008). Both the hippocampus and striatum are involved in consolidation of motor sequence memory. Neuron 58, 261-272.

11. Lohse, K.R., Wadden, K., Boyd, L.A., and Hodges, N.J. (2014). Motor skill acquisition across short and long time scales: a meta-analysis of neuroimaging data. Neuropsychologia 59, 130-141.

12. Doyon, J., and Benali, H. (2005). Reorganization and plasticity in the adult brain during learning of motor skills. Curr Opin Neurobiol 15, 161-167.

13. Penhune, V.B., and Steele, C.J. (2012). Parallel contributions of cerebellar, striatal and M1 mechanisms to motor sequence learning. Behav Brain Res 226, 579-591.

14. Penhune, V.B., and Doyon, J. (2002). Dynamic cortical and subcortical networks in learning and delayed recall of timed motor sequences. J Neurosci 22, 1397-1406. 
15. Huang, Y.Z., Rothwell, J.C., Lu, C.S., Wang, J., Weng, Y.H., Lai, S.C., Chuang, W.L., Hung, J., and Chen, R.S. (2009). The effect of continuous theta burst stimulation over premotor cortex on circuits in primary motor cortex and spinal cord. Clin Neurophysiol 120, 796-801.

16. Huang, Y.Z., Chen, R.S., Fong, P.Y., Rothwell, J.C., Chuang, W.L., Weng, Y.H., Lin, W.Y., and Lu, C.S. (2018). Inter-cortical modulation from premotor to motor plasticity. J Physiol 596, 4207-4217.

17. Gerschlager, W., Siebner, H.R., and Rothwell, J.C. (2001). Decreased corticospinal excitability after subthreshold $1 \mathrm{~Hz}$ rTMS over lateral premotor cortex. Neurology 57, 449-455.

18. Munchau, A., Bloem, B.R., Irlbacher, K., Trimble, M.R., and Rothwell, J.C. (2002). Functional connectivity of human premotor and motor cortex explored with repetitive transcranial magnetic stimulation. J Neurosci 22, 554-561.

19. Ohbayashi, M., Ohki, K., and Miyashita, Y. (2003). Conversion of working memory to motor sequence in the monkey premotor cortex. Science 301, 233-236.

20. Meehan, S.K., Randhawa, B., Wessel, B., and Boyd, L.A. (2011). Implicit sequence-specific motor learning after subcortical stroke is associated with increased prefrontal brain activations: an fMRI study. Hum Brain Mapp 32, 290-303.

21. Honda, M., Deiber, M.P., Ibanez, V., Pascual-Leone, A., Zhuang, P., and Hallett, M. (1998). Dynamic cortical involvement in implicit and explicit motor sequence learning. A PET study. Brain 121 (Pt 11), 2159-2173.

22. Boyd, L.A., and Linsdell, M.A. (2009). Excitatory repetitive transcranial magnetic stimulation to left dorsal premotor cortex enhances motor consolidation of new skills. BMC Neurosci 10, 72.

23. Meehan, S.K., Zabukovec, J.R., Dao, E., Cheung, K.L., Linsdell, M.A., and Boyd, L.A. (2013). One hertz repetitive transcranial magnetic stimulation over dorsal premotor cortex enhances offline motor memory consolidation for sequence-specific implicit learning. Eur J Neurosci 38, 3071-3079.

24. Kantak, S.S., Mummidisetty, C.K., and Stinear, J.W. (2012). Primary motor and premotor cortex in implicit sequence learning-evidence for competition between implicit and explicit human motor memory systems. Eur J Neurosci 36, 2710-2715.

25. Focke, J., Kemmet, S., Krause, V., Keitel, A., and Pollok, B. (2017). Cathodal transcranial direct current stimulation (tDCS) applied to the left premotor cortex (PMC) stabilizes a newly learned motor sequence. Behav Brain Res 316, 87-93.

26. Pollok, B., Schmitz-Justen, C., and Krause, V. (2021). Cathodal Transcranial Direct Current Stimulation (tDCS) Applied to the Left Premotor Cortex Interferes with Explicit Reproduction of a Motor Sequence. Brain Sci 11. 
27. Nitsche, M.A., Jakoubkova, M., Thirugnanasambandam, N., Schmalfuss, L., Hullemann, S., Sonka, K., Paulus, W., Trenkwalder, C., and Happe, S. (2010). Contribution of the premotor cortex to consolidation of motor sequence learning in humans during sleep. J Neurophysiol 104, 2603-2614.

28. Vidoni, E.D., and Boyd, L.A. (2007). Achieving enlightenment: what do we know about the implicit learning system and its interaction with explicit knowledge? J Neurol Phys Ther 31, 145-154.

29. Robertson, E.M. (2009). From creation to consolidation: a novel framework for memory processing. PLoS Biol 7, e19.

30. Hazeltine, E., Grafton, S.T., and Ivry, R. (1997). Attention and stimulus characteristics determine the locus of motor-sequence encoding. A PET study. Brain 120 ( Pt 1), 123-140.

31. Grafton, S.T., Hazeltine, E., and Ivry, R. (1995). Functional mapping of sequence learning in normal humans. J Cogn Neurosci 7, 497-510.

32. Oldfield, R.C. (1971). The assessment and analysis of handedness: the Edinburgh inventory. Neuropsychologia 9, 97-113.

33. Beck, A.T., Rial, W.Y., and Rickels, K. (1974). Short form of depression inventory: cross-validation. Psychol Rep 34, 1184-1186.

34. Hoddes, E., Zarcone, V., Smythe, H., Phillips, R., and Dement, W.C. (1973). Quantification of sleepiness: a new approach. Psychophysiology 10, 431-436.

35. Awiszus, F.B., J.J. (2011). TMS motor threshold assessment tool (MTAT 2.0). Brain Stimulation Laboratory. Medical University of South Carolina, USA.

36. King, B.R., Saucier, P., Albouy, G., Fogel, S.M., Rumpf, J.J., Klann, J., Buccino, G., Binkofski, F., Classen, J., Karni, A., et al. (2017). Cerebral Activation During Initial Motor Learning Forecasts Subsequent Sleep-Facilitated Memory Consolidation in Older Adults. Cereb Cortex 27, 1588-1601.

37. Rumpf, J.J., Wegscheider, M., Hinselmann, K., Fricke, C., King, B.R., Weise, D., Klann, J., Binkofski, F., Buccino, G., Karni, A., et al. (2017). Enhancement of motor consolidation by post-training transcranial direct current stimulation in older people. Neurobiol Aging 49, 1-8.

38. Janacsek, K., Shattuck, K.F., Tagarelli, K.M., Lum, J.A.G., Turkeltaub, P.E., and Ullman, M.T. (2020). Sequence learning in the human brain: A functional neuroanatomical meta-analysis of serial reaction time studies. Neuroimage 207, 116387.

39. Chen, R., Classen, J., Gerloff, C., Celnik, P., Wassermann, E.M., Hallett, M., and Cohen, L.G. (1997). Depression of motor cortex excitability by low-frequency transcranial magnetic stimulation. Neurology 48, 1398-1403. 
40. Muellbacher, W., Ziemann, U., Boroojerdi, B., and Hallett, M. (2000). Effects of low-frequency transcranial magnetic stimulation on motor excitability and basic motor behavior. Clin Neurophysiol 111, 1002-1007.

41. Maeda, F., Keenan, J.P., Tormos, J.M., Topka, H., and Pascual-Leone, A. (2000). Modulation of corticospinal excitability by repetitive transcranial magnetic stimulation. Clin Neurophysiol 111, 800-805.

42. Rizzo, V., Siebner, H.R., Modugno, N., Pesenti, A., Munchau, A., Gerschlager, W., Webb, R.M., and Rothwell, J.C. (2004). Shaping the excitability of human motor cortex with premotor rTMS. J Physiol 554, 483-495.

43. Tecchio, F., Zappasodi, F., Assenza, G., Tombini, M., Vollaro, S., Barbati, G., and Rossini, P.M. (2010). Anodal transcranial direct current stimulation enhances procedural consolidation. J Neurophysiol 104, 1134-1140.

44. Krause, V., Meier, A., Dinkelbach, L., and Pollok, B. (2016). Beta Band Transcranial Alternating (tACS) and Direct Current Stimulation (tDCS) Applied After Initial Learning Facilitate Retrieval of a Motor Sequence. Front Behav Neurosci 10, 4.

45. King, B.R., Rumpf, J.J., Heise, K.F., Veldman, M.P., Peeters, R., Doyon, J., Classen, J., Albouy, G., and Swinnen, S.P. (2020). Lateralized effects of post-learning transcranial direct current stimulation on motor memory consolidation in older adults: An fMRI investigation. Neuroimage 223, 117323.

46. Chen, J., McCulloch, A., Kim, H., Kim, T., Rhee, J., Verwey, W.B., Buchanan, J.J., and Wright, D.L. (2020). Application of anodal tDCS at primary motor cortex immediately after practice of a motor sequence does not improve offline gain. Exp Brain Res 238, 29-37.

\section{Declarations}

\section{Authors' contributions}

Felix Psurek

acquired, analyzed and interpreted the data; wrote the first draft of the manuscript

Bradley R. King interpreted the data; revised the manuscript for intellectual content

Joseph Claßen designed the study; revised the manuscript for intellectual content

Jost-Julian Rumpf designed and conceptualized study; analysed and interpretated the data; revised and edited the manuscript 


\section{Data Availability}

The data used to support the findings of this study are available from the corresponding author upon reasonable request.

\section{Competing Interests}

All authors declare that they have no competing interests as defined by Nature Research, or other interests that might be perceived to influence the results and/or discussion reported in this paper.

\section{Funding Statement}

No industry, government or institutional funding was received for this research.

\section{Figures}

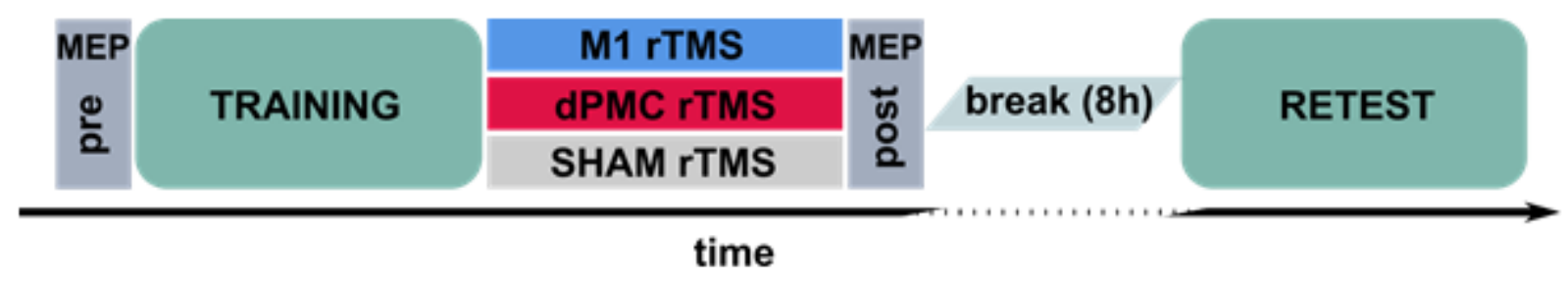

\section{Figure 1}

Experimental design. At the onset of the experiment, 20 motor evoked potentials (MEPs) of the right abductor pollicis brevis muscle (APB) were evoked at $0.1 \mathrm{~Hz}$ with the stimulator output set to an individually adjusted intensity to elicit MEPs of $\sim 1 \mathrm{mV}$ (MEPpre). This baseline assessment of corticospinal excitability (CSE) was followed by 14 blocks of task training with the right hand (TRAINING). Immediately after termination of TRAINING, participants received low-frequency $(1 \mathrm{~Hz})$ repetitive transcranial magnetic stimulation (rTMS) of either the left primary motor cortex (M1 rTMS), the dorsal premotor cortex (dPMC rTMS), or SHAM rTMS. 20 MEPs from the APB were again recorded (MEPpost) with the same individually adjusted stimulator output level as during the MEPpre assessment. Participants were retested on task performance ( 14 blocks) after an interval of 8 hours (RETEST). 


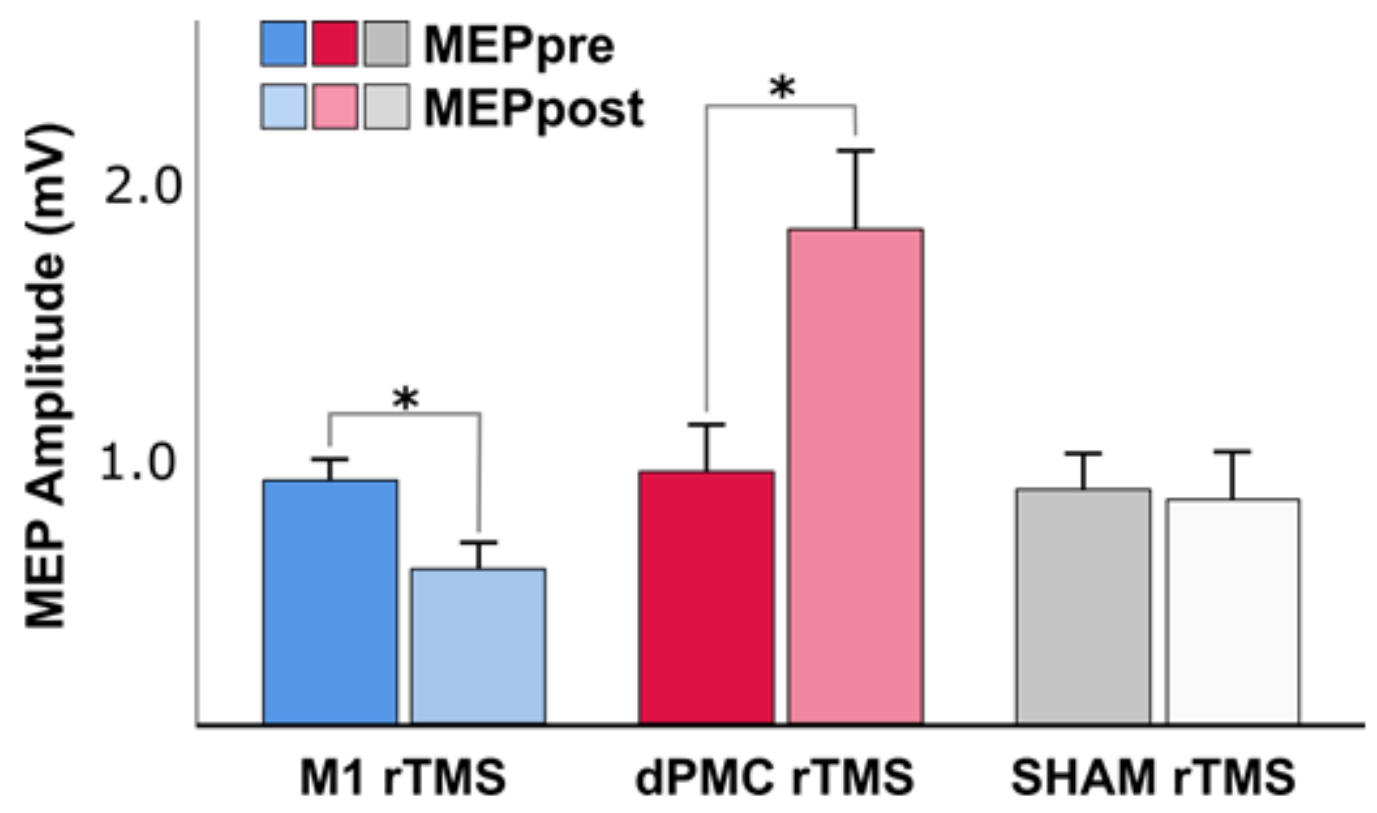

Figure 2

$1 \mathrm{~Hz}$ rTMS-induced effects on corticospinal excitability. Mean amplitudes of 20 motor evoked potentials (MEPs) before the training session (MEPpre) and immediately after (MEPpost) 20 minutes of posttraining $1 \mathrm{~Hz}$ rTMS of the primary motor cortex (M1 rTMS), the dorsal premotor cortex (dPMC rTMS), or sham rTMS. Error bars indicate the standard error of the mean. The asterisks indicate significant differences between the MEPpre and MEPpost assessments, $p<0.05$. 
A
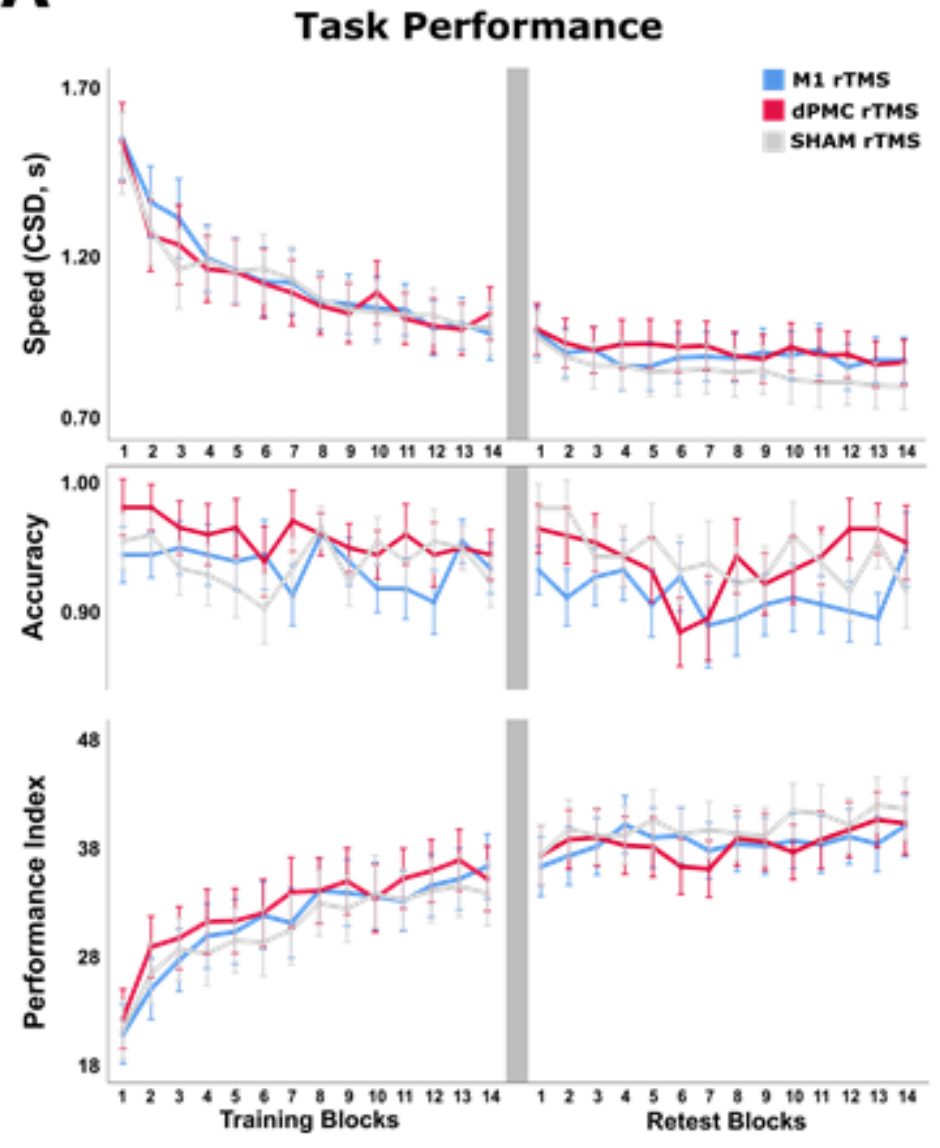

B
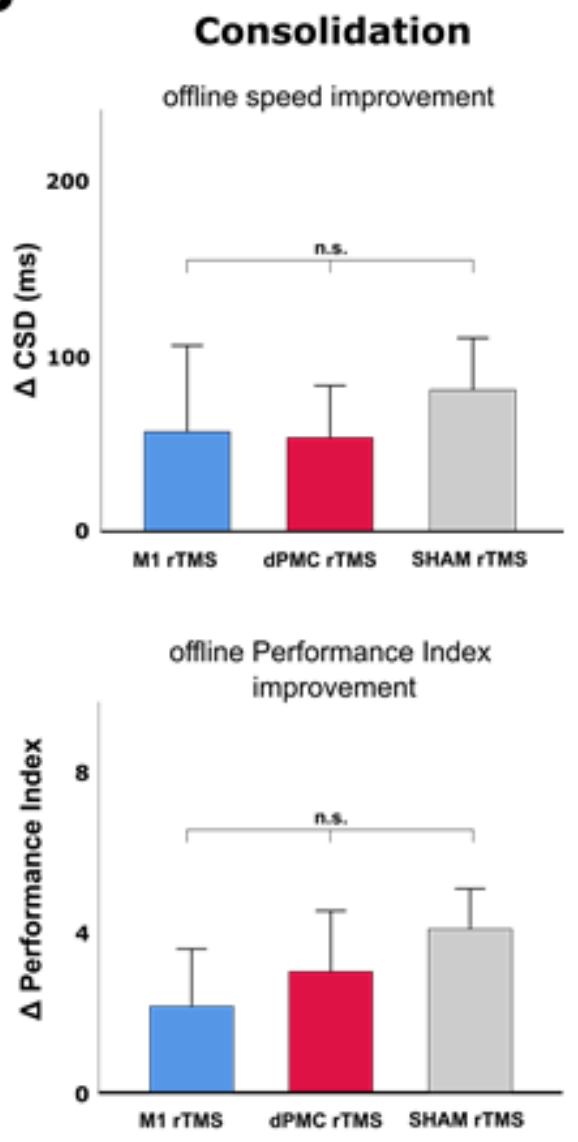

\section{Figure 3}

Behavioural results. (A) Performance changes of all three experimental groups (post-training M1 rTMS, dPMC rTMS, and SHAM rTMS) across blocks of task execution in terms of speed (mean duration of a correct sequence, CSD), accuracy (ratio of the number of correct sequences per block/number of maximum correct sequences per block), and the combined performance index. The grey column between the training and retest graphs represents the 8-hour break interval. Error bars indicate the standard error of the mean. (B) Offline task performance changes (difference) between end-of-training (mean performance across the last 4 blocks of the training session) and beginning of the delayed retest (first block of delayed retesting). Note that differences were computed such that positive values indicate offline performance improvements (i.e., offline learning). Error bars indicate the standard error of the mean (n.s.: not significant). 